\title{
One-Dimensional Meso-Structures: The Growth and the Interfaces
}

\author{
Lisheng Huang $1,2,3$, Yinjie Su${ }^{2}$ and Wanchuan Chen ${ }^{1}$ \\ ${ }^{1}$ Department of Physics, National Cheng Kung University, Tainan, \\ ${ }^{2}$ National Laboratory of Solid State Microstructures, Nanjing University, Nanjing, \\ ${ }^{3}$ College of Sciences \& College of Materials Science and Engineering, \\ Nanjing University of Technology, Nanjing, \\ 1Taiwan \\ 2,3 China
}

\section{Introduction}

One-dimensional (1D) meso-structures have become the focus of intensive research worldwide due to their unique physics and potential to revolutionize broad areas of device applications. They act as the most basic building blocks of nano-electronic systems, nano-optics and nanosensors, so the controlled growth of these meso-structures is important for applying them in these fields. Materials properties can be tuned through control of micro-structural characteristics such as the physical size, shape, and the surface. Efforts to explore structures with multiple length scales unite the frontiers of materials chemistry, physics, and engineering. It is in the design and characterization of advanced materials that the importance of new interdisciplinary studies may be realized [1-4]. Recent research focused on well-faceted mesostructures has shown that the shape as well as the hetero-[5, 6] or homo-junctions $[7,8]$ contribute much to the tuning of properties of structured materials. Many significant properties, including optical, chemical, as well as electronic, have been revealed to be shape- or junction-related. For example, the lasing behaviors of nonlinear optical nano-scale wires or belts derive from the resonance cavity effect functioned by the parallel end-faces of the nanostructures [9-11]. Quantitative characterization of optical waveguiding in straight and bent nanowires is achievable in active devices [12]. Such study has shown that the optimization of surfaces, boundaries, and interfaces in materials with well-faceted structures plays an important role in furthering the application of these materials.

For efficient fabrication and assembly of well-faceted meso-structures, the anisotropy of the crystal can be utilized to control the nucleation and manipulate the surface energy [13, 14]. Macroscopically, a crystal has different kinetic parameters for different crystal planes guided by certain growth conditions. After initial nucleation, a crystallite will commonly develop into a three-dimensional entity with well-defined, low index crystallographic facets. Thus, the growth anisotropy can be advantageously utilized to create crystals with specific desired characteristics through control of the growth conditions. It has been extremely successful in different growth systems, such as solution-based route for growing shaped nanocrystals, vapor-phase growth of quasi-one-dimensional meso-structures with well- 
defined cross sections and surface polarities as well as some other exotic configurations through vapor-liquid-solid (VLS) or vapor-solid (VS) process [15-20].

In this chapter, we have examined the growth mechanisms and the morphology evolutions of one-dimensional meso-structures systematically based on the experimental and theoretical aspects of crystal growth. The 1D ZnO meso-structures will be selected as an example to show the morphologic evolution at multiple length scales. The quasi-onedimensional $\mathrm{SnO}_{2}$ meso-structures are studied to describe the morphological multiformity of crystal growth. The outline of the chapter is as follows. In Sect. 2, the growth of $\mathrm{ZnO}$ meso-structures is discussed, which includes the controlled growth (Sect. 2.1), structural characterization and crystal models (Sect. 2.2), the growth process and mechanism (Sect. 2.3), and structure-related optical properties (Sect. 2.4). In Sect. 3, the $\mathrm{SnO}_{2}$ zigzag mesostructures growth mechanism is discussed, which includes the controlled growth (Sect. 3.1), structural characterization and crystal models (Sect. 3.2), the morphological evolution mechanism (Sect. 3.3). Concluding remarks are given in Sect. 4.

\section{ZnO meso-structures}

\subsection{Growth control}

$\mathrm{ZnO}$, a wide direct band-gap semiconductor, is piezoelectric and transparent to visible light [21]. It is attracting much attention for application in UV light-emitters, varistors, transparent high power electronics, surface acoustic wave devices, piezoelectric transducers, gas-sensors, photo-catalysts, and as a window material for display and solar cells [22-31]. The wurtzite structure of the $\mathrm{ZnO}$ crystal has pronounced anisotropy. It possesses three fast growth directions of $\langle 2 \overline{1} \overline{1} 0\rangle,\langle 01 \overline{1} 0\rangle$, and $\langle 0001\rangle$. Currently much effort has been focused on the fabrication of $\mathrm{ZnO}$ nano-/micro-scale structures. A number of methods, based on solid reaction, solution based synthesis, and vapor rout have been developed to grow this material. These methods include the reaction of zinc salt with base, thermal decomposition, pulse laser deposition (PLD), thermal evaporation/vapor phase transport (CVD), metal-organic CVD, molecular beam epitaxy (MBE), electrochemical deposition, chemical bath deposition, aqueous solution decomposition, modified micro-emulsion, and sol-gel methods [32-44]. ZnO nano-/micro-structures of varied geometries, exemplified by wires/rods, belts/ribbons, comb-like structures, tetra-pod whiskers and their various assemblages have all been produced by our group (Figs. 1a-d).

We have also reported a new type of modulated and well-faceted $\mathrm{ZnO}$ microfibers, which was synthesized via a convenient CVD process [8]. Considering the decomposition of of $\mathrm{Ni}\left(\mathrm{NO}_{3}\right)_{2}$ at high temperature, we used nickel oxide $\left(\mathrm{Ni}_{2} \mathrm{O}_{3}\right)$ as a catalyst. This proved to be an efficient way for growing the modulated microfibers. Fig. 2 shows a typical SEM morphology of the as-synthesized product. It is evident that the products are composed of microfibers with periodic junctions at a significant percentage (over 95\%) of the yield and over $80 \%$ reproducibility from run to run.

The fibers with very thin junctions usually grow parallel to each other, and the roots appear to be compressed and broad. The lengths of the fibers typically range in between 200 and $500 \mu \mathrm{m}$. the longest one observed was nearly a millimeter. The spacing between two neighboring junctions normally ranges from 5 to $30 \mu \mathrm{m}$. The side surfaces of the fibers are well-faceted. Note that the V-shaped junction derives from the concavo-concave morphology, and the angle between the left and the right facets is exactly of 60 or 120 
degrees. The fiber is characteristically decorated by periodically prism-like junction arrays. We refer to this structure as a "junction-prism" structure.
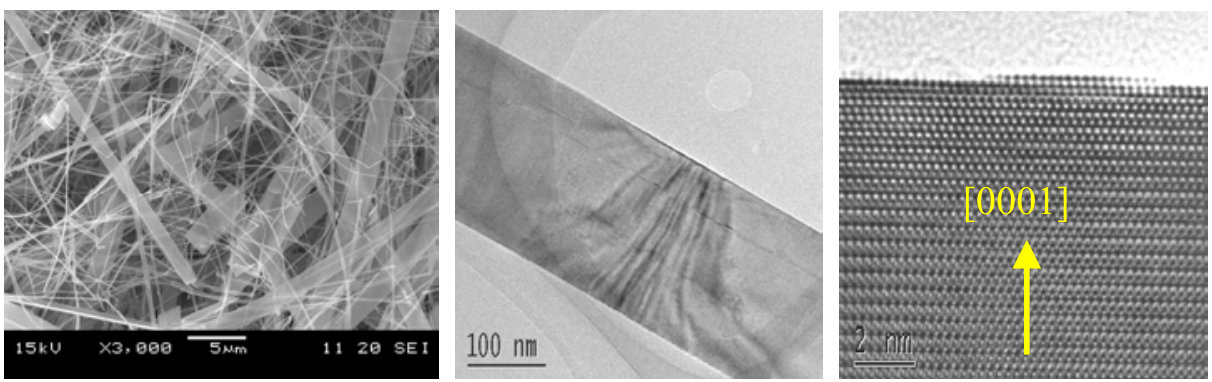

(a)
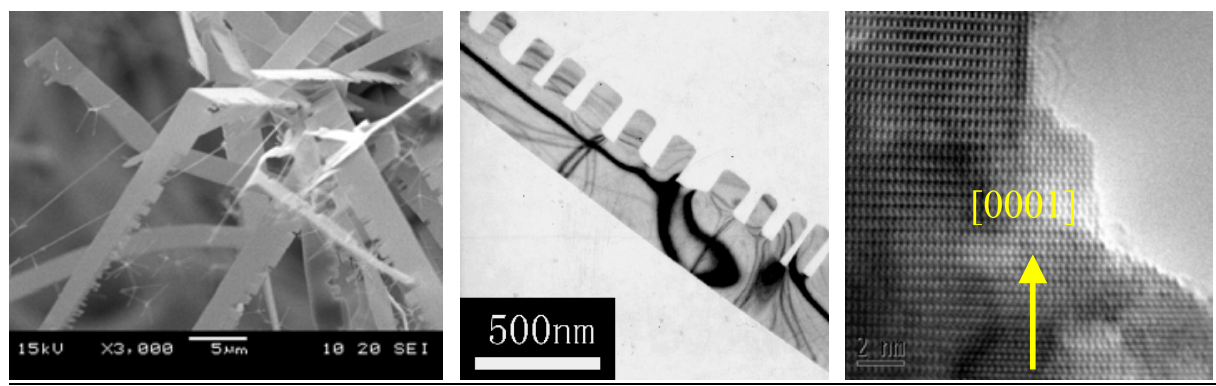

(b)
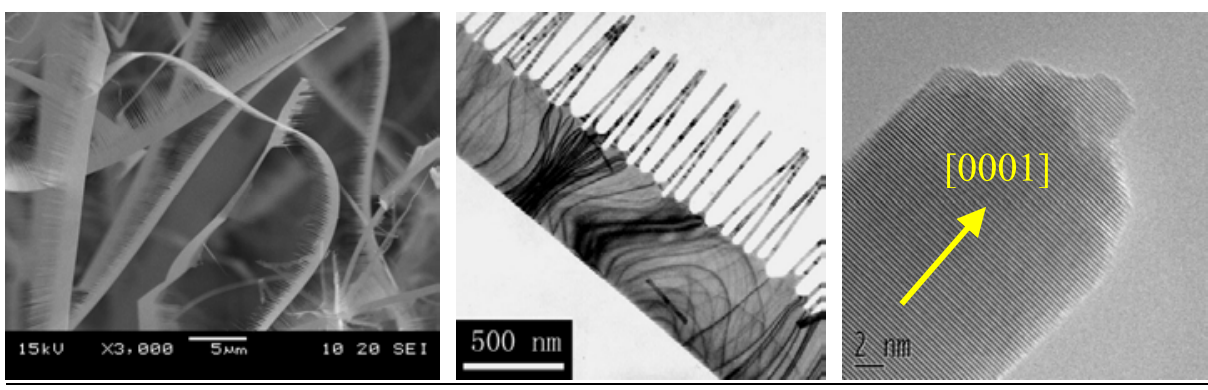

(c)
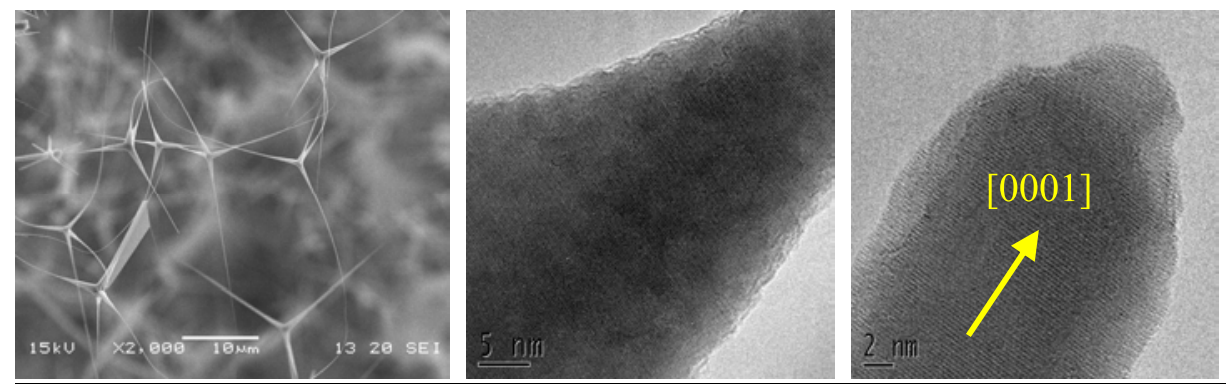

$(\mathbf{d})$

Fig. 1. ZnO meso-structures of belts (a), comb-like structures (b, c) and tetra-pod whiskers (d). 


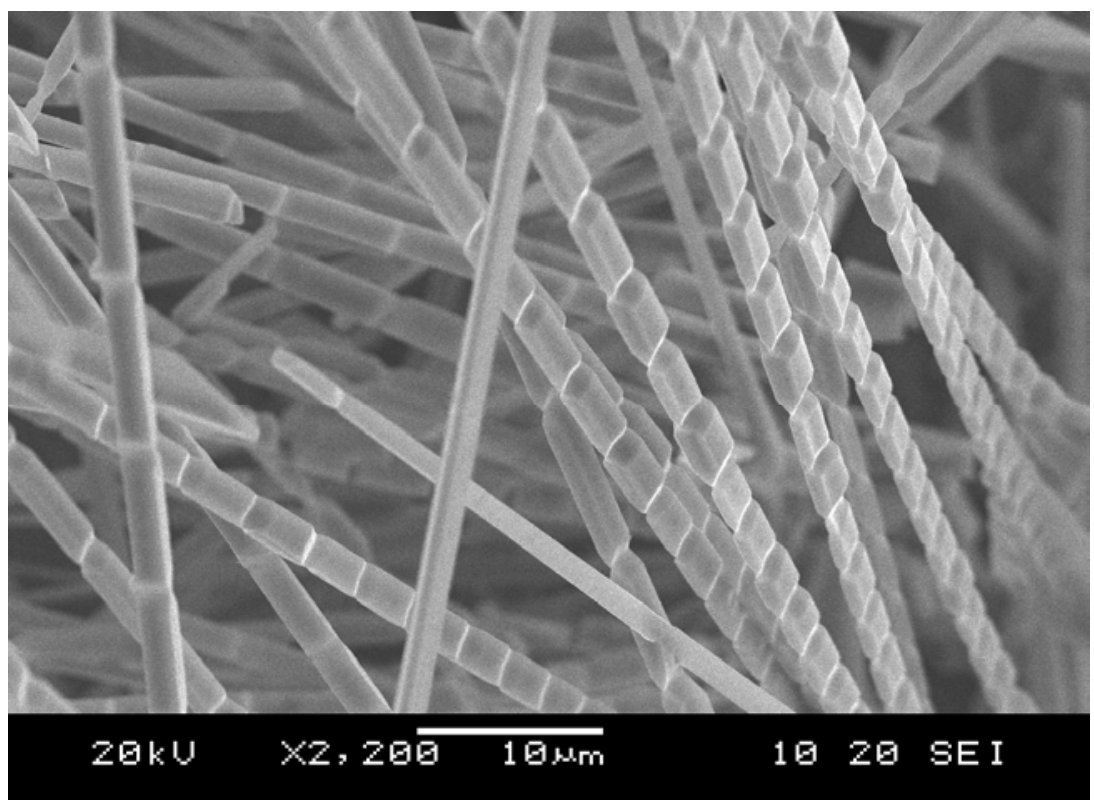

Fig. 2. ZnO junction-prism structure.

\subsection{Structural characterization and crystal models}

XRD and EDS measurements were performed for element analysis and phase determination. XRD studies show a typical wurtzite structure of $\mathrm{ZnO}$ with cell constants of $\mathrm{a}=0.324 \mathrm{~nm}$ and $\mathrm{c}=0.519 \mathrm{~nm}$ (Fig. 3a, JCPDS No. 36-1451). EDS studies (equipped in TEM) at the head, junction and root of a fiber show only peaks belonging to $\mathrm{Zn}$ and $\mathrm{O}$ without any other impurities (Figs. 3b-d).

In our studies, the presence of a small amount of nickel oxide is critical to synthesizing these modulated and well-faceted $\mathrm{ZnO}$ fibers. Although the vapor-liquid-solid (VLS) crystal growth mechanism explains the catalysis growth of some microstructures, no element nickel was observed found in our samples. Of course, the possibility exists that the quantities may be less than can be measured by XRD or EDS analysis. It is likely that the role of the nickel is the same as that of indium oxide and lithium carbonate for nanoring growth [45]. While no catalysts added for their growth, we believe that another important intrinsic factor for growth of the modulated microstructures is the intense anisotropy of the wurtzitestructured $\mathrm{ZnO}$ along different axes.

Crystallographic orientations of the fibers were obtained by EBSD. Fig. 4a shows the microfiber with a flat facet upturned, which was automated EBSD mapped for the selected area (Fig. $4 \mathrm{~b}$ ). As EBSD requires a highly tilted surface (near $70^{\circ}$ tilt), several microfibers were searched until one was found to give indexable EBSD patterns with illumination corresponding to a flat surface tilted to $70^{\circ}$. The map displayed is corrected for the $70^{\circ}$ tilt whereas the SEM image is not tilt-corrected. Pole figures obtained from the EBSD map data show the [2 $\overline{11} 0]$ direction aligned with the growth direction, the broad surfaces parallel to 
$\{0001\}$ plane and the side surfaces parallel to $\{01 \overline{1} 0\}$ (Fig. 4c). A schematic unit cell displayed in the orientation was obtained by EBSD (Fig. 4d). The growth direction of the microfiber is [2 $\overline{110} 0$ ( $a$ axis) and the side surfaces are \pm (0001). The broad top and bottom surfaces are parallel $\{01 \overline{1} 0\}$ planes.

It was found there are two types of oriented fibers in the production. Fig. 5a shows the crystal models. This is consistent with the crystal structure of the $\mathrm{ZnO}$. The wurtzite structure of the $\mathrm{ZnO}$ crystal has pronounced anisotropy, it possesses three fast growth directions of $\langle 2 \overline{11} 0\rangle,\langle 01 \overline{1} 0\rangle$, and $\langle 0001\rangle$. Generally, [0001] is the fastest based on the kinetic mechanism involved. [0001] growth minimizes the area of exposed $\{0001\}$ faces (Fig. $5 b)$. Under thermodynamic equilibrium conditions, the surface energy of the polar $\{0001\}$ planes is larger than that of the nonpolar planes of $\{01 \overline{1} 0\}$ or $\{2 \overline{11} 0\}$. Moreover, the surface energies differ less between the $\{01 \overline{1} 0\}$ and $\{2 \overline{1} \overline{1} 0\}$ planes. Fig. $5 \mathrm{c}$ illustrates the basic configurations evolved from $\mathrm{ZnO}$ hexagonal unit in Fig. 5b. Changing the growth condition to activate various growth facets, microstructures would be synthesized in shapes with higher complexity than those of the familiar wire, rod, belt, and sphere-like structures. Thus, it is often found that the produced well-faceted $\mathrm{ZnO}$ fibers with periodic junction-prisms preferentially grow along [01 $\overline{1} 0]$ as opposed to the [2110] direction. The structure model shown in Fig. 5a (insert) illustrates a [0110] preferred growth axis of the fiber and the geometric relationships between all its outer facets.
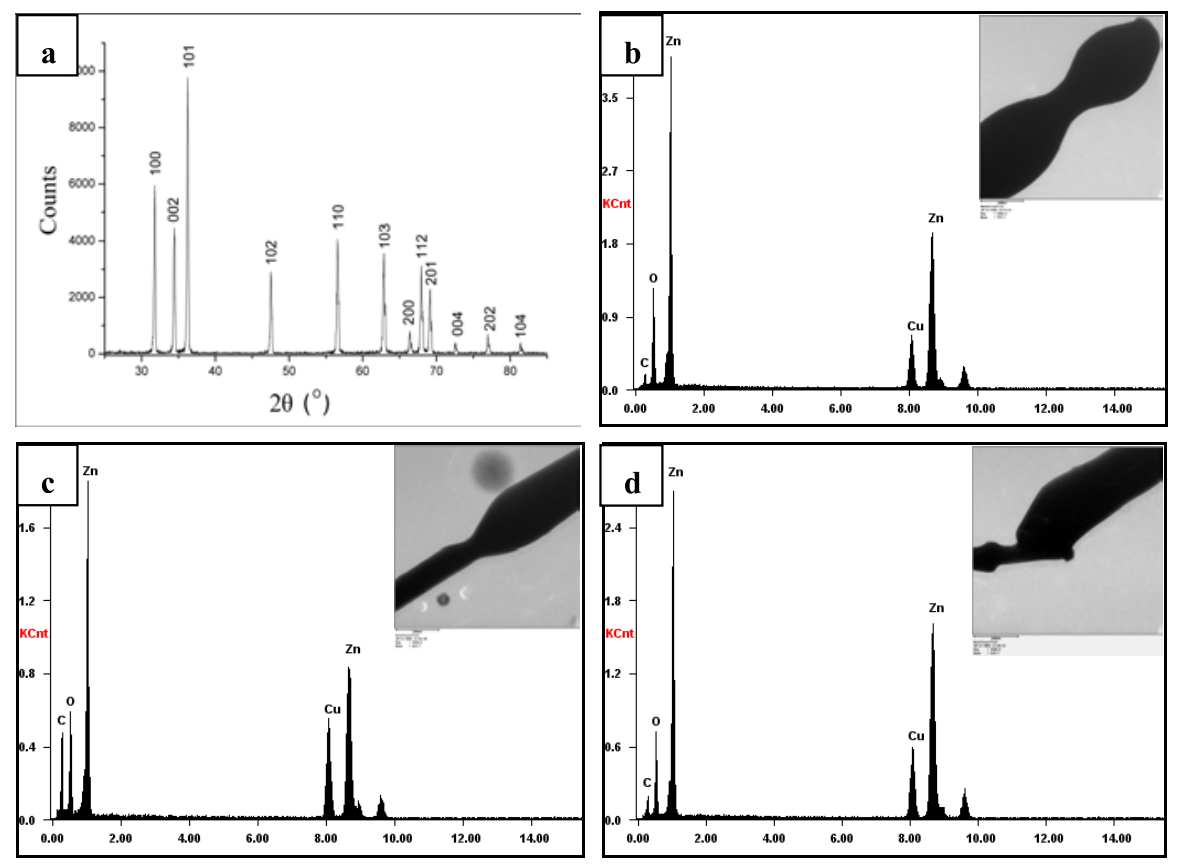

Fig. 3. XRD pattern showing wurtzite structure of ZnO (a), EDS studies at the head, the junction and the root of a fiber $(b-d)$. 


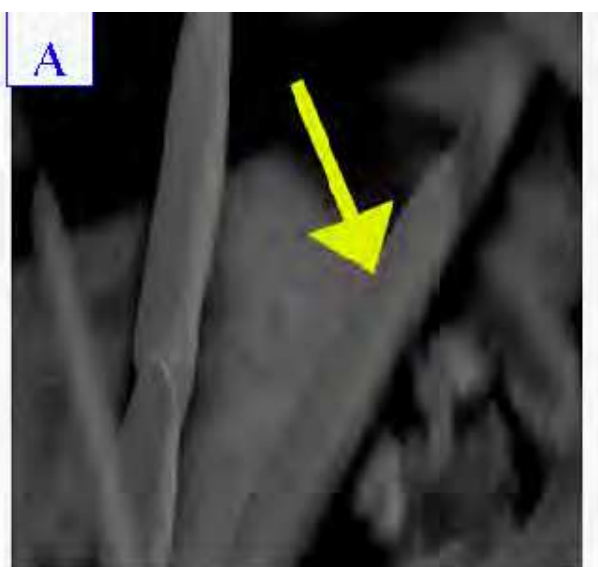

$2 \mu \mathrm{m}$

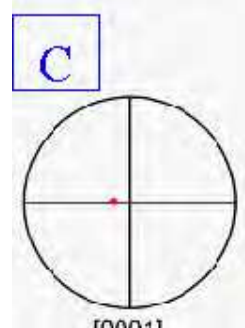

[0001]

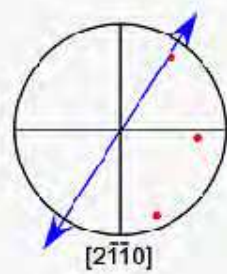

[2110]
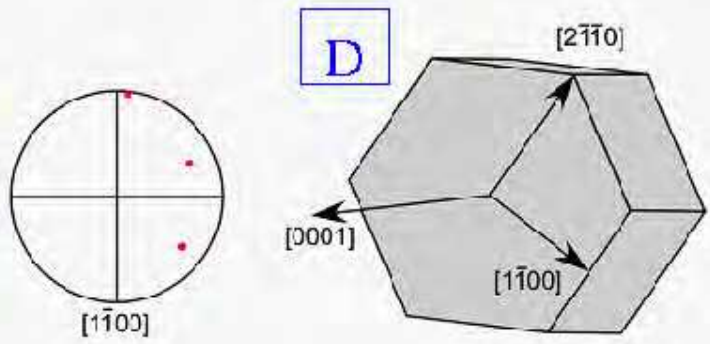

Fig. 4. EBSD measurement for a microfiber.

\subsection{Growth process and mechanism}

SEM investigations on the microfibers demonstrate growth mechanism of the junctionprisms structures. Fig. 6a shows a newly growing head of a fiber, the growth unit of a nearly hexagonal prism is grown perpendicularly on the nanobelt base. Presumably, the small growth head would develop anisotropically and contact the adjacent unit forming a junction (Fig. 6b). Some similar configurations have been studied by our group [46]. We found that $\mathrm{ZnO}$ nano/microcombs are kin to these fibers: every tooth could be considered as a segment unit of the fiber. If the teeth grow short in the [0001] direction and thick in diameter, then they would contact each other and the morphology should be identical to these junction-like fibers (Fig. 6c, d). Additional experiment also showed that large amount necklace-shape structures could be produced. Every microstructure consisted of a row of rhomboids that are equally separated on a straight base of a narrow nanobelt (Fig. 6e, 6f). An anisotropic growth process is shown as follows: a nanobelt base was formed by fast catalysis growth along [2110] or [0110], followed by slow growth along [0001], forming the separate units of nearly hexagonal prisms. Developing on the nanobelt, these small units merged and formed junctions.

Some SEM images of the fibers show segment units quenched at different stages of their growth, and careful examination of the unit's morphology gives insight into the growth 
process. Fig. 7 (right panel) shows typical units in various stages of growth, along with their schematics (left panel). Although the SEM images are of different fibers, it is presumed that each fiber undergoes a similar sequence of steps during the growth process.
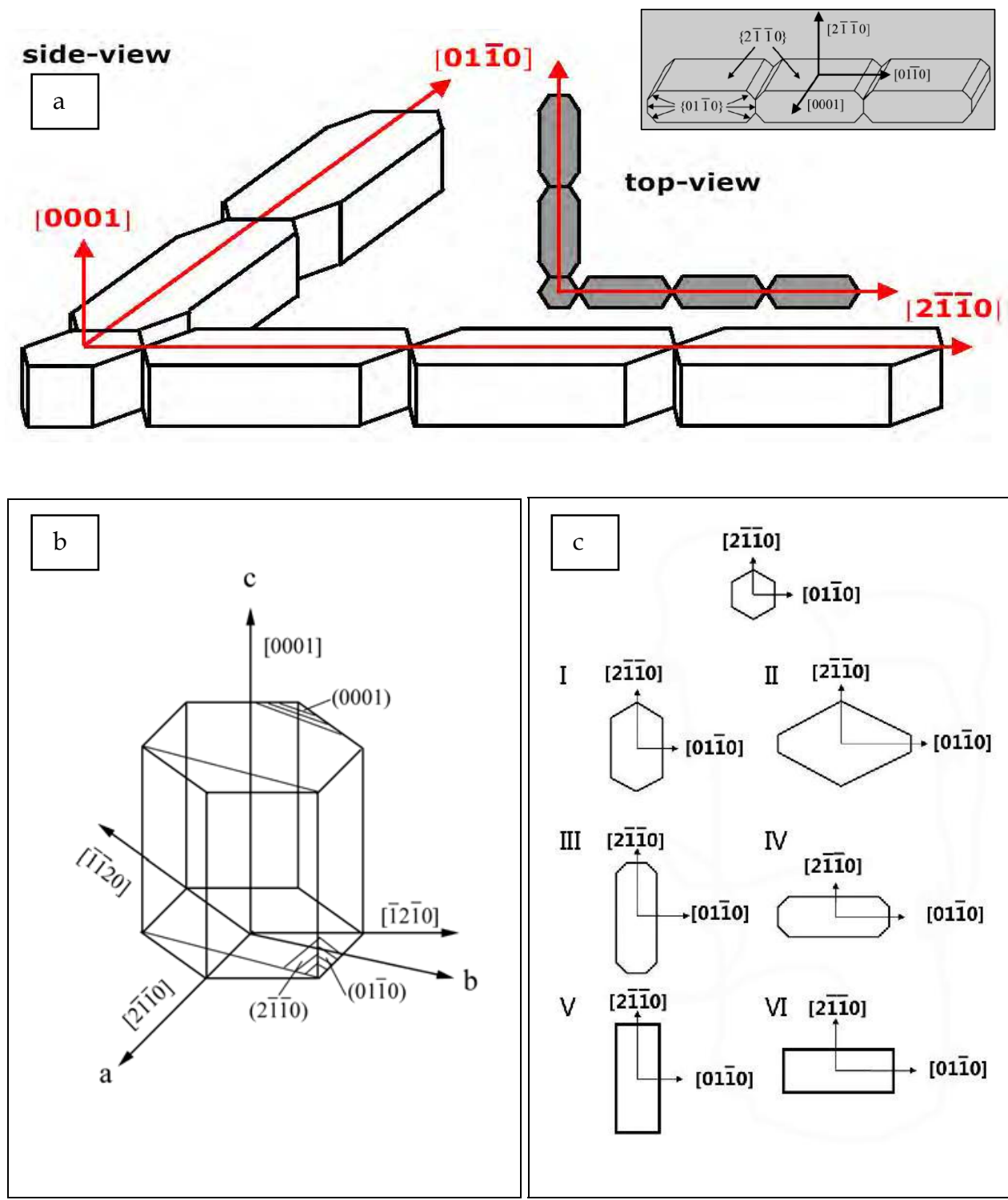

I

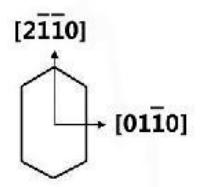

III

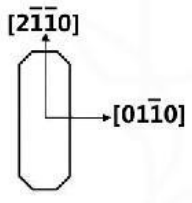

V

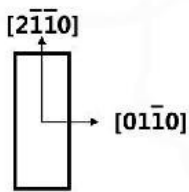

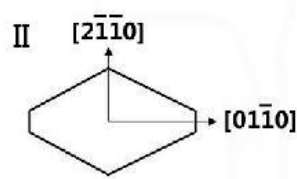

IV

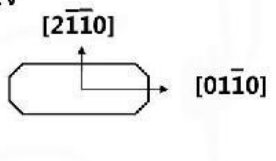

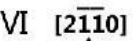

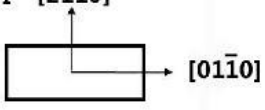

Fig. 5. (a) crystal mode of the two types of oriented $\mathrm{ZnO}$ fibers, (b) crystal mode of wurtzite structure of $\mathrm{ZnO}$, (c) the basic configurations evolved from $\mathrm{ZnO}$ hexagonal unit. 


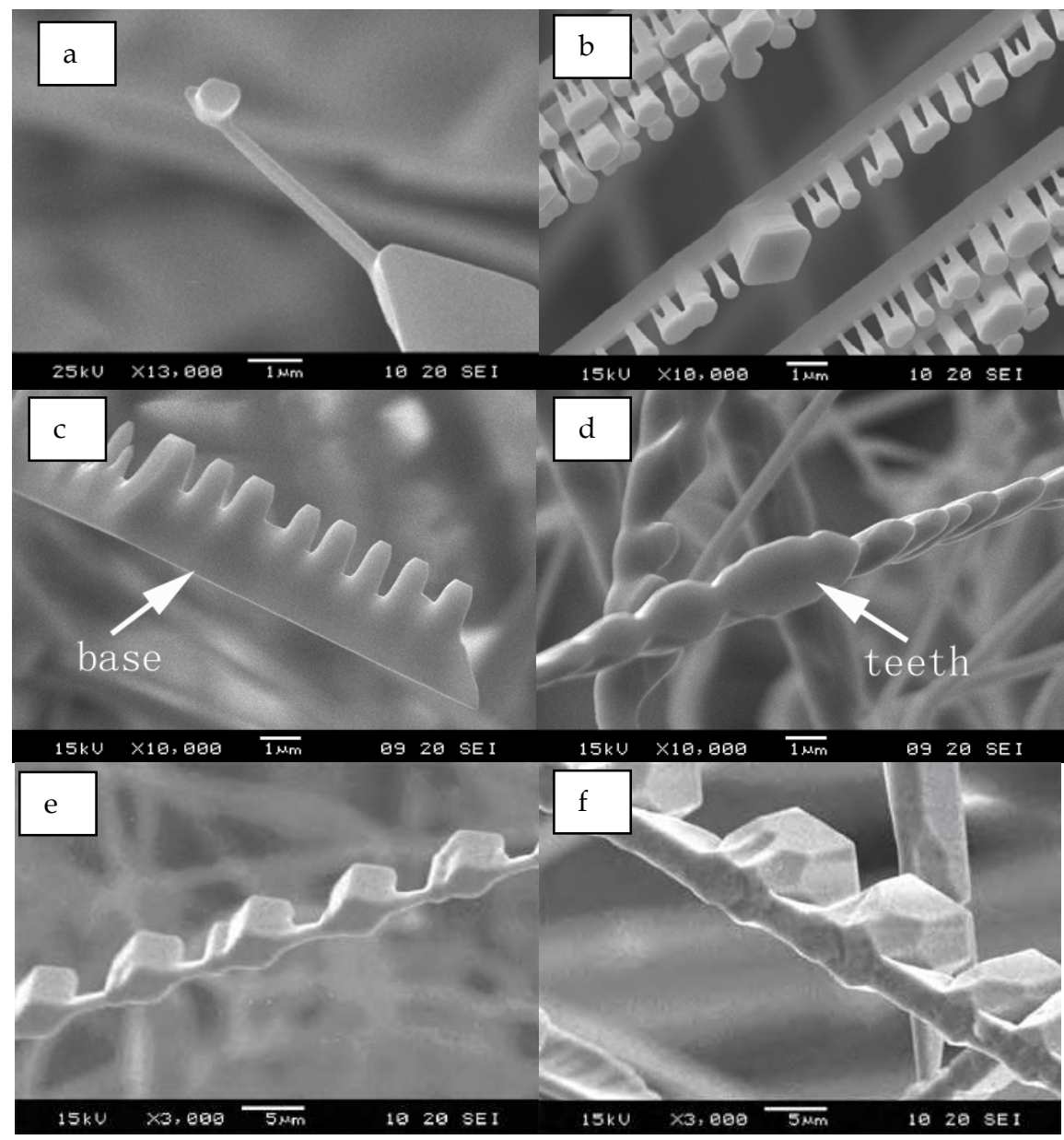

Fig. 6. (a) a newly growing head of a fiber, (b) small growth heads would develop anisotropically and contact the adjacent unit forming a junction, (c, d) $\mathrm{ZnO}$ micro-combs are kin to these fibers, $(e, f)$ necklace-shape structures.

For example, consider the growth of [01̄0] oriented fibers:

1. The first step is to grow a [01 0 ] oriented base with $\{2 \overline{1} \overline{1} 0\}$ side surfaces and top surfaces of $\{0001\}$. Subsequent grow will be self-modulated by nucleation and growth of the epitaxial pyramids on the c-face, (0001), of the base. The separate units growing along the c-axis on the base have hexagonal shapes (Fig. 7a, left panel are the crystal models). The six sided surfaces are equivalent planes of $\{01 \overline{1} 0\}$.

2. The units constructing a regular fiber with periodic junctions exhibit a prolonged eightsquare shape, where the four profile $\{01 \overline{1} 0\}$ faces are partly exposed, and two broad $\{2 \overline{11} 0\}$ faces neighbor them (Fig. 7b). This evolution could be explained by an enhanced growth along [01̄̄0] (the base growth direction) and a confined epitaxy perpendicular to [01 $\overline{1} 0]$. 

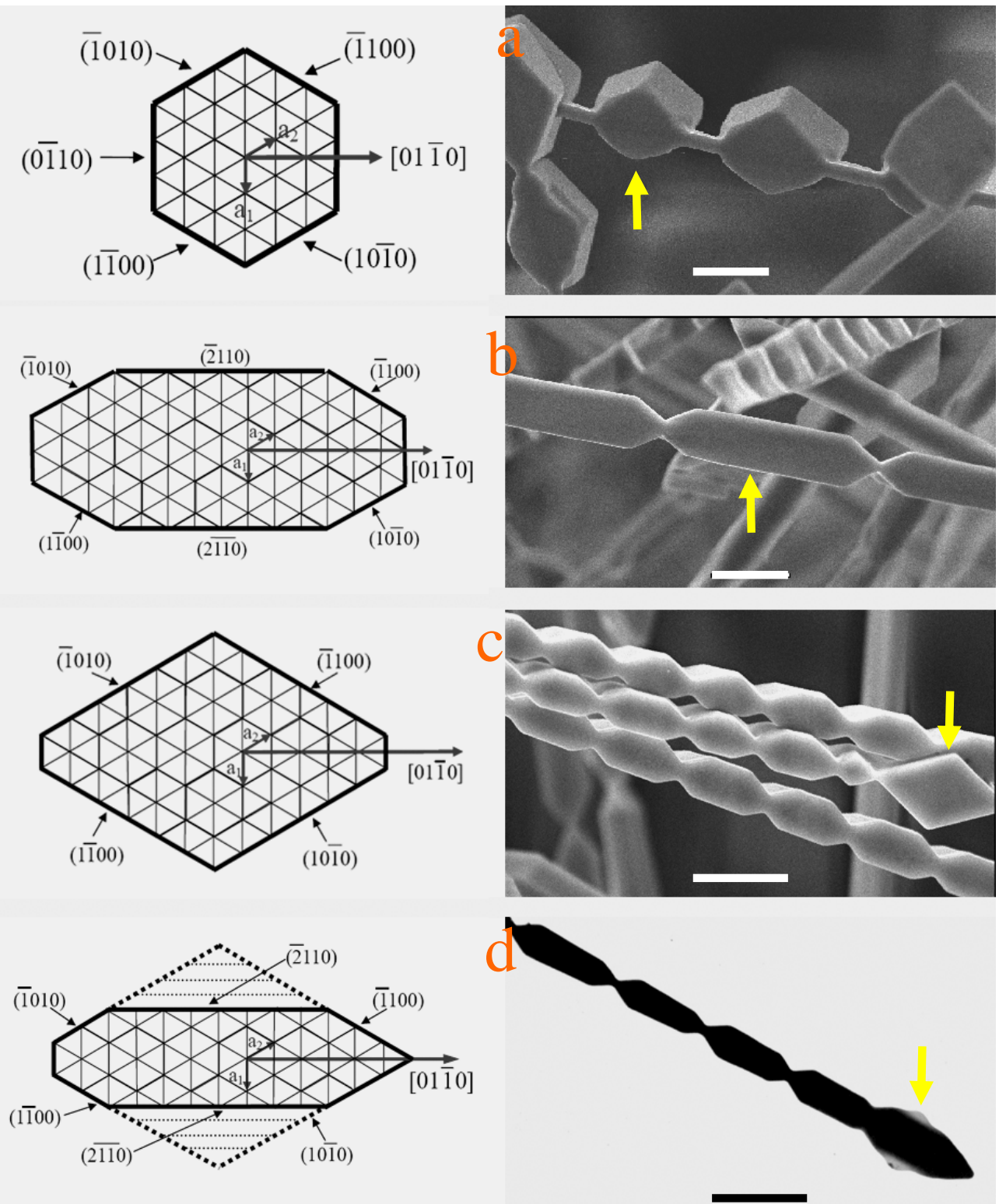

Fig. 7. Typical units in various stages of growth along with their schematics. 
3. A prolonged hexagonal head of a fiber shows evidence for enhanced growth along [01̄̄0] (Fig. 7c).

4. TEM observations show that the anti-confined epitaxy process is perpendicular to $[01 \overline{1} 0]$. Thin epitaxial layers are growing on the two broad $\{2 \overline{11} 0\}$ facets, and the growth would cease once the four $\{01 \overline{1} 0\}$ side surfaces are completely grown out of existence and the two $\{2 \overline{11} 0\}$ facets disappear (Fig. $7 \mathrm{~d}$ ).

Thus, we can deduce that two-step anisotropic growth as well as the confinement effect of the base (substrate) could result from these modulated and well-faceted junction-like fibers. A schematic representation of the growth process for the modulated fiber is illustrated in Fig. 8. Note that in the practical growth process, the base of the fiber has been combined into one united body with the segment units, but sometimes one side of the fiber is thicker than that of the other side. This is evident in the contrast in SEM images (Fig. 2). In order to grow very regular segment units, the growth condition should be controlled.

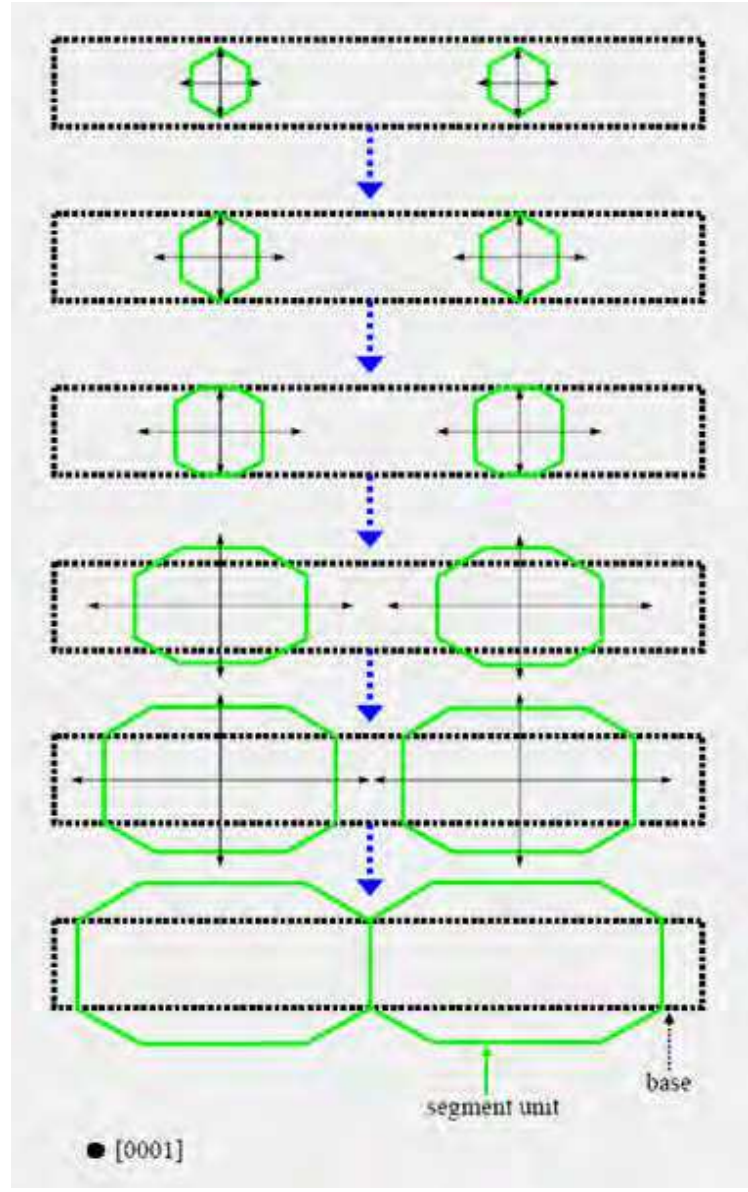

Fig. 8. A schematic representation of the growth process for the modulated fiber. 


\subsection{Structure-related optical properties}

Because it approximately undergoes a thermodynamically equilibrium during the growth process of the fibers, all facets of a fiber are commonly low index crystallographic faces. According to the growth process and the crystal models, all the side surfaces of $\{01 \overline{1} 0\}$ and $\{2 \overline{1} 10\}$ should have coordinative crystalline qualities. $\mu$-Raman studies further proved this point of view. The Raman spectra obtained at the $\{01 \overline{1} 0\}$ facets of a junction and at the $\{2 \overline{11} 0\}$ facets on the stem are shown in Fig. 9 (the inset shows the sample), respectively. No apparent difference is observed between the spectra from the junction facets and the stem facets.

Since the wurtzite structure of $\mathrm{ZnO}$ belongs to the $C_{6 v}^{4}$ space group, the zone center optical phonons are: $A_{1}+2 E_{2}+E_{1}$ [47]. In the spectra, two Raman active $E_{2}$ modes were observed at 101 and $437 \mathrm{~cm}^{-1}$, and four Raman active modes-- $\mathrm{A}_{1}$ and $\mathrm{E}_{1}$ transverse (TO), at 380 and 407 $\mathrm{cm}^{-1}$, and longitudinal (LO), at 574 and $583 \mathrm{~cm}^{-1}$, with second order vibrations observed at 208,334 and $1050-1200 \mathrm{~cm}^{-1}$. These results can be entirely explained on the basis of the $\mathrm{ZnO}$ crystal [48], and signify the good crystalline properties of the junction stem facets of a fiber.

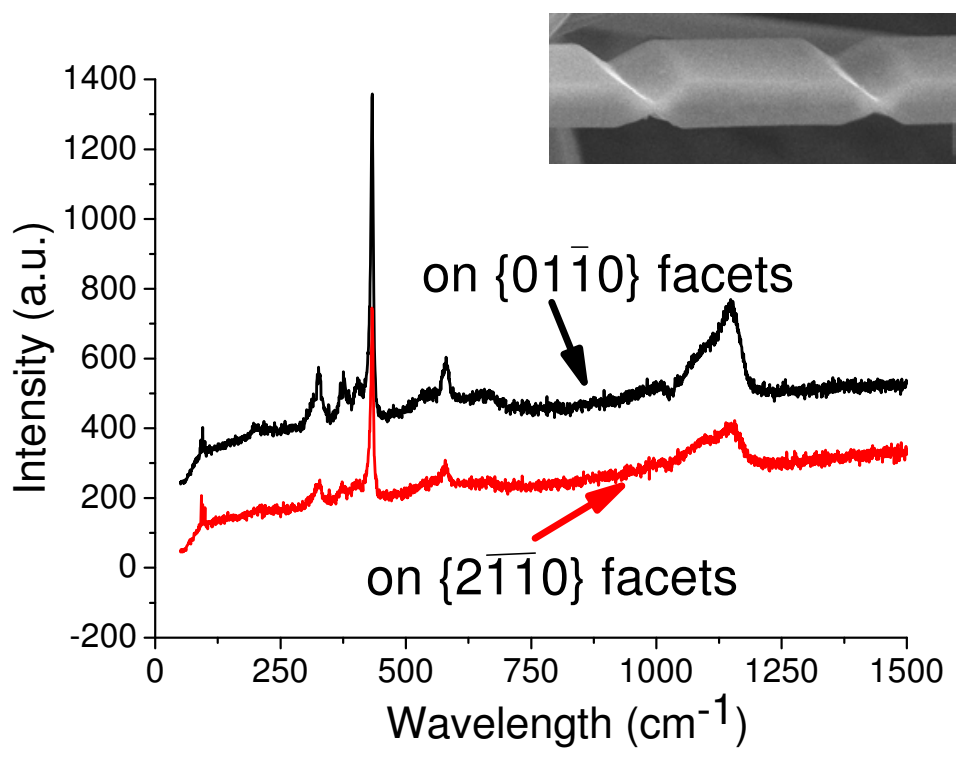

Fig. 9. Raman spectra obtained at the [01 $\overline{1} 0]$ facets of a junction and at the $\{2 \overline{11} 0\}$ facets on the stem.

Room temperature micro-PL spectra shown in Fig.10 indicate the enhancement of the green light emission at the junction. The spectrum obtained from the part between the two junctions consists of an intensive UV peak at $383 \mathrm{~nm}$ and a weak green band around $510 \mathrm{~nm}$. The spectrum around the junction indicates that the green band is strong. This was further demonstrated using a PL microscope. The PL microscopy images show the fibers emitting strong green light at the junctions (Fig. 11h). 


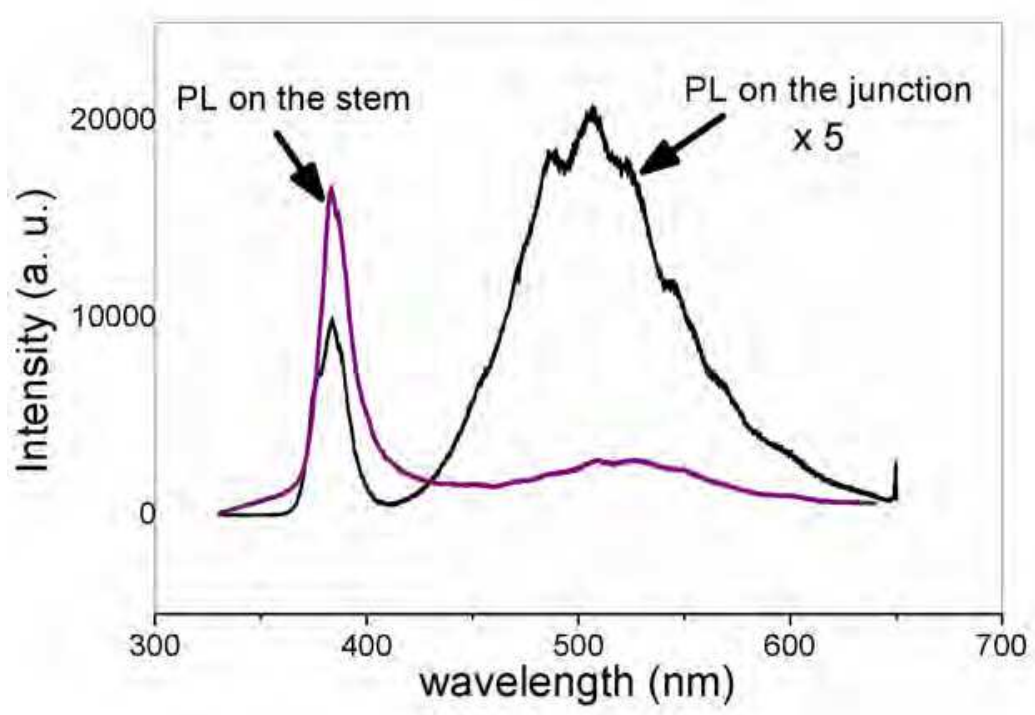

Fig. 10. Room temperature micro-PL spectra on the stem and at the junction.

It is generally accepted that the UV peak at $383 \mathrm{~nm}$ resulted from free excitonic emission of $\mathrm{ZnO}$ [49], while the green band arises from the recombination of a shallowly trapped electron within a deeply trapped hole [50]. Note that two neighboring units form one thin junction, the V-like slots upon/below the junctions are not suitable as a platform (substrate) for uniform epitaxial growth of the crystals, thus the intrinsic defects such as oxygen vacancies easily develop, resulting in the enhancement of the green light emission [51]. However, the further results of fluorescence microscopy suggest that the inhomogeneous PL emission of green light along the fiber stem, which is characterized by the periodic enhancement at the nearly isometric junctions, should be mainly attributed to the waveguide property of the well-faceted fibers.

The produced well-faceted $\mathrm{ZnO}$ fibers with periodic junctions preferentially grow along

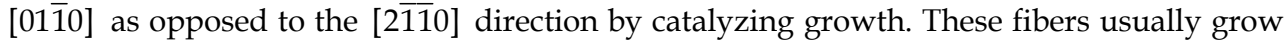
broad roots, and the bottom surfaces are $(000 \overline{1})$ (Fig. 11a). When the fibers were dispersed onto the quartz substrates by drop-casting, most of the fibers attach to the substrate with broad $\{0001\}$ facets (Fig. 11c and 11e), the fragments without broad roots (Fig. 11b) attach with $\{2 \overline{11} 0\}$ facets (Fig. 11d and 11f).

These natural junction-prism arrays as well as the well-faceted surfaces associated with the transparent and homogeneous nature of crystalline $\mathrm{ZnO}$ medium offer sharp interfaces between $\mathrm{ZnO}$ and air (or other media) for guiding the propagation of light effectively. The optical morphology of the fiber shown in a typical barcode-like black-bright contrast (Fig. 11d) was imaged with a transmission optical microscope. Note in the experimental setup, the parallel light used to illuminate the sample in the microscope came from a lamp underlying the sample, while the camera was located atop the sample. The dark contrast 
regions correspond to the junction-prisms, while the bright contrast regions correspond to the building blocks of the fiber, which are separated by the junctions. This typical optical phenomenon suggests that refraction and reflection are strongly modulated by the junctionprism arrays within this structural fiber. When parallel light propagates perpendicular to the boundary between the $\mathrm{ZnO}$ crystal $\left(\mathrm{n}_{\mathrm{ZnO}} \approx 2\right)$ and air medium $\left(\mathrm{n}_{\mathrm{air}} \approx 1\right)$, it splits into two parts: light transmitted into $\mathrm{ZnO}$ and the light reflected back into air. Considering the reflection and transmission coefficients of $\mathrm{ZnO}$ crystal, about $88 \%$ of the incident light was refracted. Moreover, because the $\mathrm{ZnO}$ crystal is optically denser than air, no light should enter the air from the $\mathrm{V}$-shape surfaces of the upper junction-prism, where the entire incident light was reflected back due to total reflection.
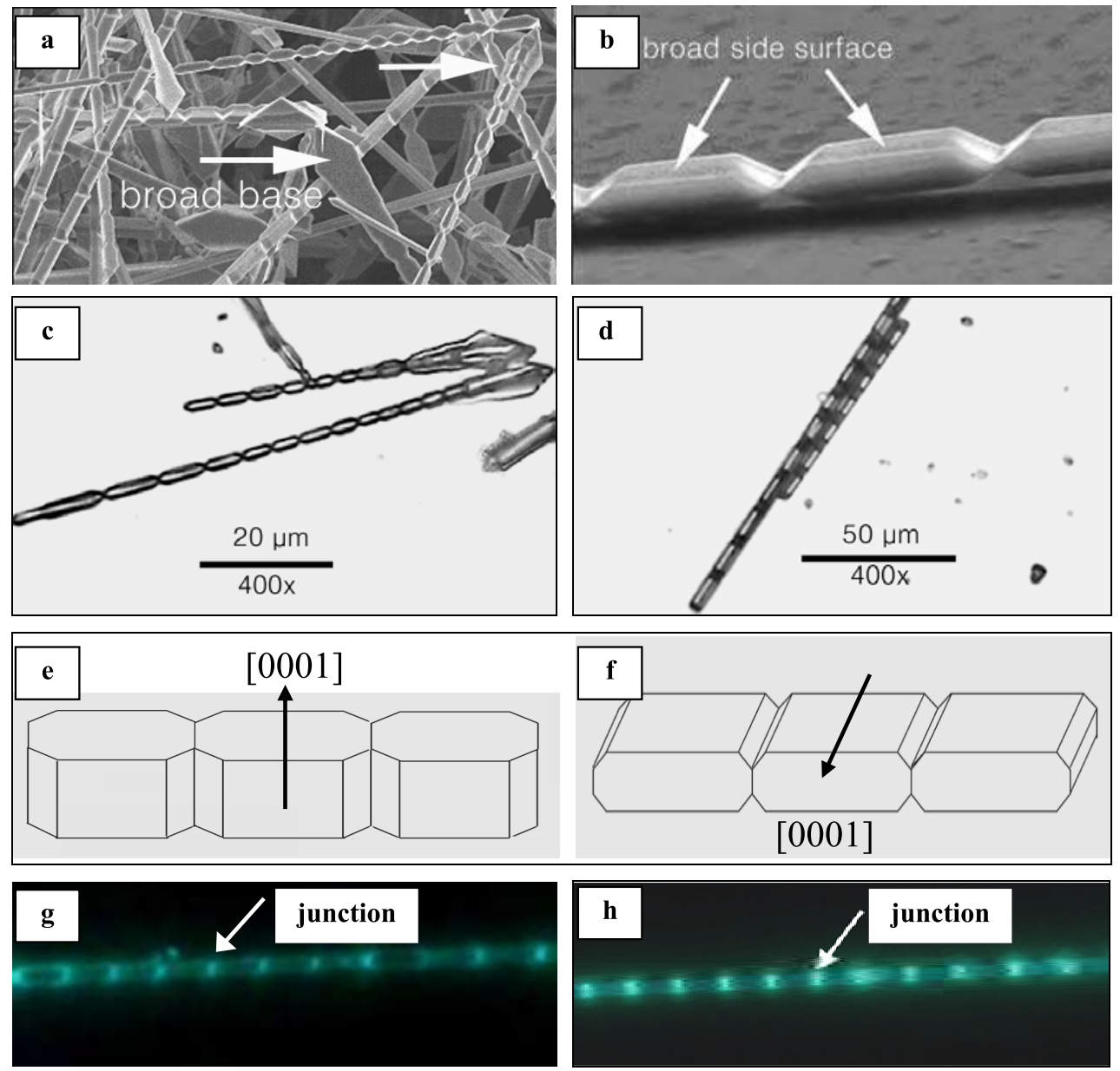

Fig. 11. Most of the fibers attach to the substrate with broad $\{0001\}$ facets (a, c, e and g), other fragments without broad roots attach with $\{2 \overline{11} 0\}$ facets $(b, d, f$ and $h)$. 
When the fibers were excited by UV light (wavelength: $325-380 \mathrm{~nm}$ ) with a fluorescence microscope, it is interestingly found that the enhanced green light emits the periodic junctions (Fig. 11h). This result could be explained by the optical waveguide behavior of the well-faceted structure with the junction-prism arrays of $\mathrm{ZnO}$. As to the side surfaces of single building blocks of a fiber, every two parallel broad $\{2 \overline{11} 0\}$ surfaces and two narrow $\{0001\}$ surfaces could serve as a natural square cavity/waveguides (Fig. 12a). In general, the $\mathrm{Vo}^{*}$ centers contributing to the defect-related green emission should be present at the surface region of a given $\mathrm{ZnO}$ crystal [50]. An ideal model elucidates the featured enhancement of the green light emitting at the junction-prisms. Analyzing one of these $\mathrm{Vo}^{*}$ centers, its emitting light is easily reflected by the two $\{2 \overline{11} 0\}$ surfaces along the z-axis (i.e., [0170]). Note Fig. 12b-1, if the fiber is uniform and cuboid in shape, it should be an ideal bar-like waveguide and the emitting light from the total-reflection widows would be sent out from the ends of the fiber due to total reflection. In this case, it can be considered as an ideal optical fiber. However, the junction-prism arrays of the present fibers destroy the totalreflection condition (Fig. 12b-2). Thus, the junction-prism arrays change the propagation paths of the emitted light and most of the light from the total-reflection windows is guided out of the junction-prism regions directly, resulting in enhanced illumination at the junctions. Moreover, even if the emitted light goes straight though a junction-prism, it would encounter the next junction, and be sent out at last. The thicker the junction of a fiber, the more easily light is arrested by the junction-prism (Fig. 12b-3). All these observations show that the periodic junction-prisms, which provide emitting windows for intrinsic emissions, naturally tune the guided light in the well-faceted fibers.

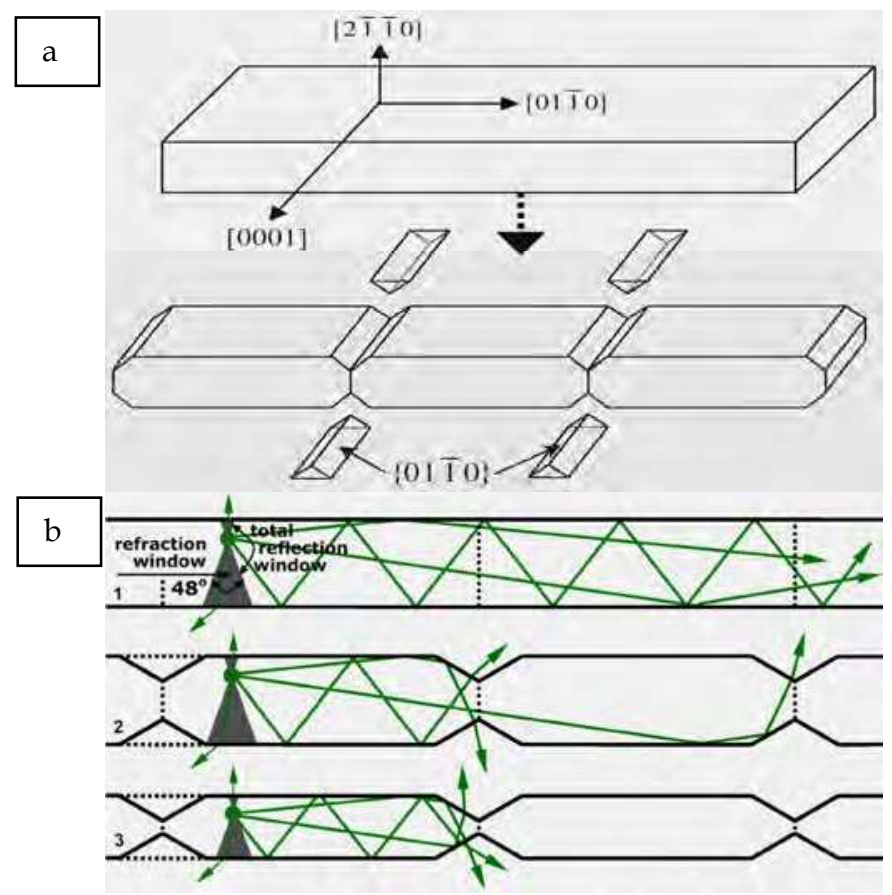

Fig. 12. Schematic illustrations of light reflection at the surface of the junction-prism structure. 


\section{$3 \mathrm{SnO}_{2}$ zigzag shaped meso-structures}

\subsection{Controlled growth}

$\mathrm{SnO}_{2}$ has been paid attention in a variety of applications in chemical, optical, electronic and mechanical fields, due to its unique high conductivity, chemical stability, gas sensitivity and semiconducting properties [52]. Many syntheses of $\mathrm{SnO}_{2}$ with different morphologies, such as nano-scale belt, wire, disk and dendrite, have been reported [53-56]. Herein, we report on a kinetics-controlled method to realize selective growth of $\mathrm{SnO}_{2}$ unconventional zigzag shaped fibers. The morphological evolution process was investigated via SEM and TEM. Previously, the method used to grow $\mathrm{SnO}_{2}$ single crystals is the high temperature gas phase reaction of evaporating $\mathrm{SnO}_{2}$ or $\mathrm{SnO}$ to lead to $\mathrm{SnO}$ in the gas phase, and subsequent reoxidation [57]. Here we used a lower temperature decomposition of SnO solid powders to produce $\mathrm{Sn}$ vapor for deposition, and then to oxidize it to $\mathrm{SnO}_{2}$. In order to selected deposition of structured products, the growth kinetics was controlled [58].

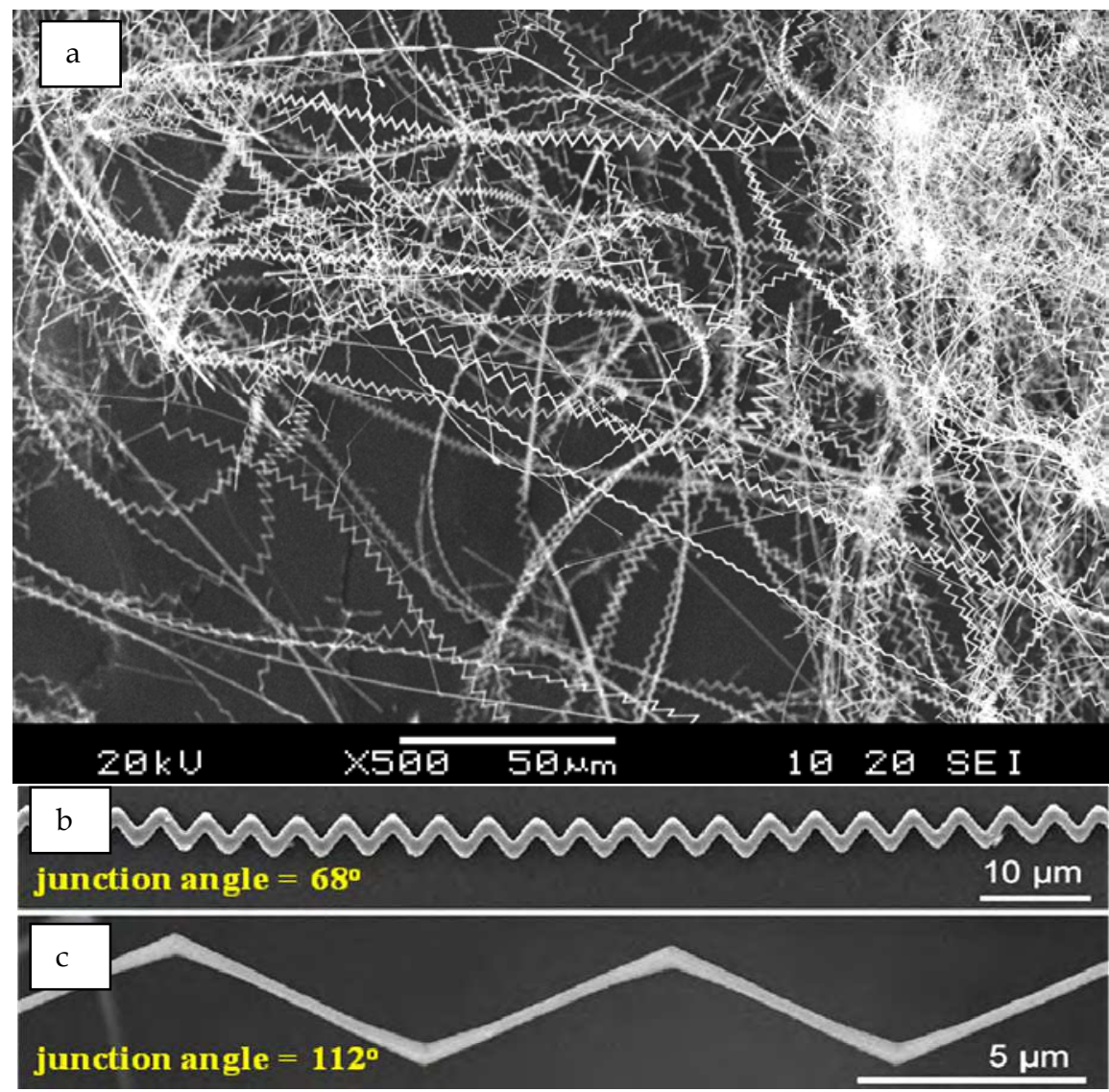

Fig. 13. (a) SEM image of the high yield $\mathrm{SnO}_{2}$ zigzag fibers, the zigzags with junction angles of about $68^{\circ}(\mathrm{b})$ and $112^{\circ}(\mathrm{c})$, respectively. 


\subsection{Structural characterization and crystal models}

The SEM image shows the typical growth of zigzag fibers as that shown in Fig. 13a. XRD results show the both structured products are with same crystallography structure: tetragonal rutile $\mathrm{SnO}_{2}$. The high yield zigzags extend very long and collide with each other. The typical space of one zigzag period ranges from 2 to $10 \mu \mathrm{m}$, and the transverse swing is in the range of 5 to $10 \mu \mathrm{m}$. The length of the zigzag increases with the growth time, sometimes it can be up to several millimeters. In addition, there are more than three types of the angles of the zigzag junctions. Most of them are about $68^{\circ}$ (Fig. 13b), and a few are approximately $112^{\circ}$ (Fig. 13c), 90 and 124으.

The TEM images (Fig. 14a, 14b) give insight of a zigzag angle of $68^{\circ}$. Electron diffractions on the entire junction and on the two blocks reveal that the zigzag is single crystal. The growth directions of the two blocks are parallel to the crystallographic equivalent directions of [101] and [101] , respectively. High-resolution TEM images (Fig. 14c, 14d) indicate the entire fiber has same lattice arrangements. The structural models are illustrated in Fig. 14b. Structurally, the $\pm[101]$ and $\pm[10 \overline{1}]$ in tetragonal $\mathrm{SnO}_{2}$ are equivalent directions. The angle between the [101] and $[10 \overline{1}]$ directions and that between the $[\overline{1} 0 \overline{1}]$ and [ $\overline{1} 01]$ is $68^{\circ}$, while the angle

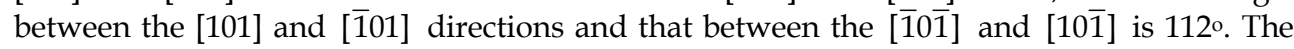
experimental results of about $68^{\circ}$ and $112^{\circ}$ correlate well with these values. The formation of a zigzag is mainly accomplished though repeated alternation of growth orientations between the $\pm[101]$ and $\pm[10 \overline{1}]$. Namely, a zigzag could be separated into two types of building blocks, which laterally combine each other periodically. The zigzags with other junction angles should repeatedly shift its growth directions along some other low-index directions, such as from [101] to [001].

\subsection{Morphological evolution}

Careful examination of the zigzag's morphologies gives insight into the growth habits. As that shown in Fig. 15a, we usually found thin fiber has narrow slab-like morphology with sharp junction corners. The top/down surfaces of the building blocks could be indexed as $\pm(010)$ planes, and the side surfaces are $\pm(10 \overline{1})$ and $\pm(101)$, alternately. After further growth, the morphologies of the sample would become well-faceted with some new $\pm(100)$ facets present opposite to the junctions (Fig. 15b, 15c). Although the states are of different fibers, it can be presumed that each fiber undergoes a similar sequence of steps during the morphological evolution. The zigzag fiber would be formed by a two-step growth process. The first step is to fast grow to finalize the zigzag frame; the second step is to laterally grow to thicken its diameter. The evolution process illustrated in Fig. 15d reflects the lateral thickening process. In the beginning, the vapor species favor deposition at the V-like slots and it results in some atom steps (Fig. 15e), and then the new arrived species continuously arrange at the steps parallel to the side surface. The epitaxies would cease once the arrange layers meet the ridges of the junctions, due to the higher energy there. At last, these homoepitaxies equally thicken a fiber in width and some $\pm(100)$ facets are constructed at the same time. Note that the transverse swing of the fiber does not change all along, and the original zigzag frame decides the final frame $\left(\mathrm{A}_{0}=\mathrm{A}_{\mathrm{f}}\right)$. The longer the growth time, the more $(100)$ surface area is present. Ideally, the final morphology could be predicted to be a rectangular crystal bar with long axis parallel to [001] direction and enclosed by lower energy planes of $\pm(010)$ and $\pm(100)$. This evolution tents to minimize the surface free energy, so the growth should seek thermodynamical equilibrium and be mainly dominated by surface free energy. 
Based on this argument, we can explain why fewer zigzags with $112^{\circ}$ angels contrast against the zigzags with $68^{\circ}$ angels in the products. If the zigzag growth changes from [101] to [101] periodically, some higher energy facets of $\pm(001)$ would be constructed after the lateral thickening process. Thus it is not favorable from the energy point of view.
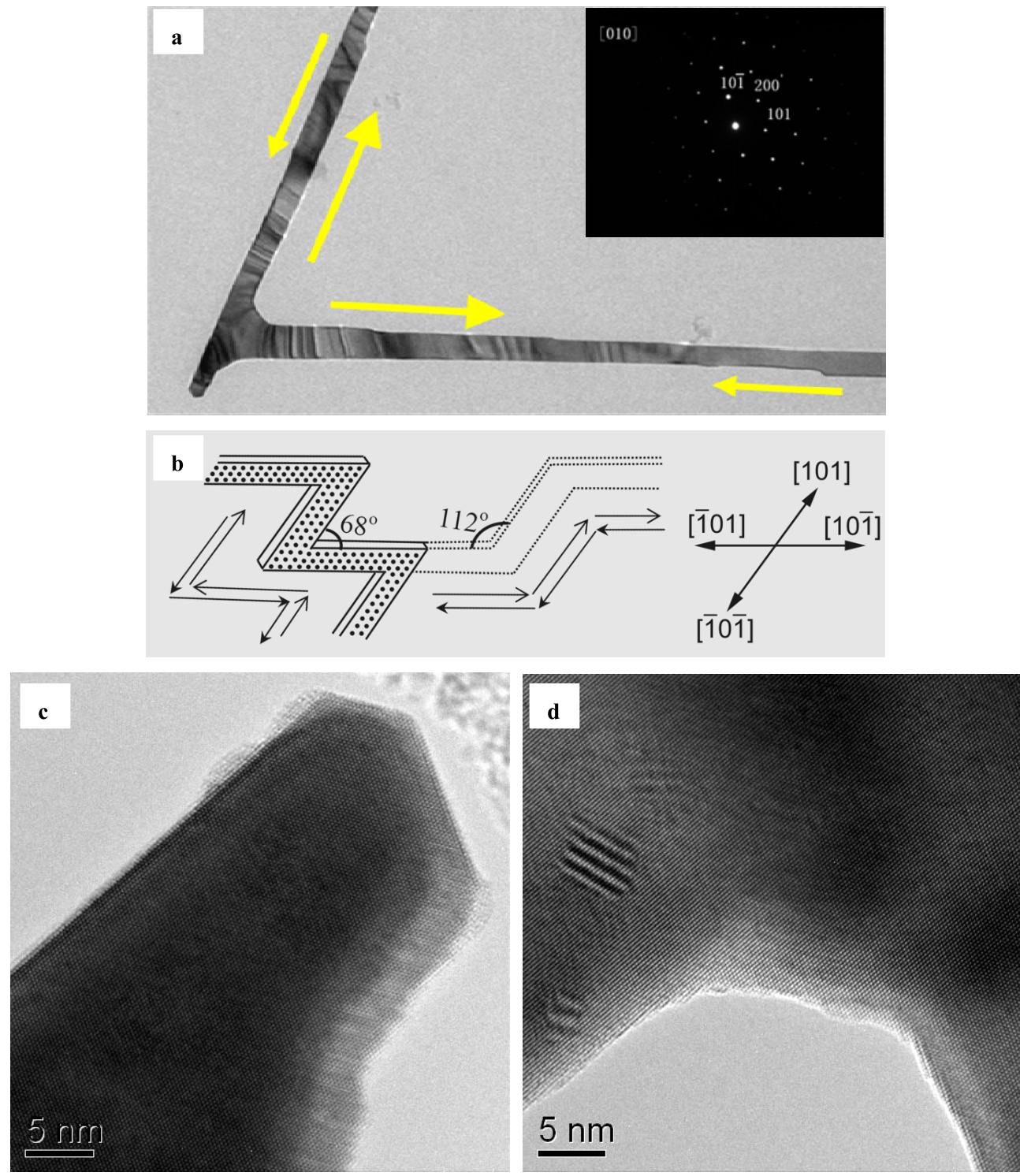

Fig. 14. (a) TEM image showing the junction angles of about $68^{\circ}$, the electron diffractions on the two blocks showing single crystal nature and $<101>$ orientations, (b) crystal structural models for the zigzags, (c, d) high-resolution TEM images taken on the head and V-shape areas. 

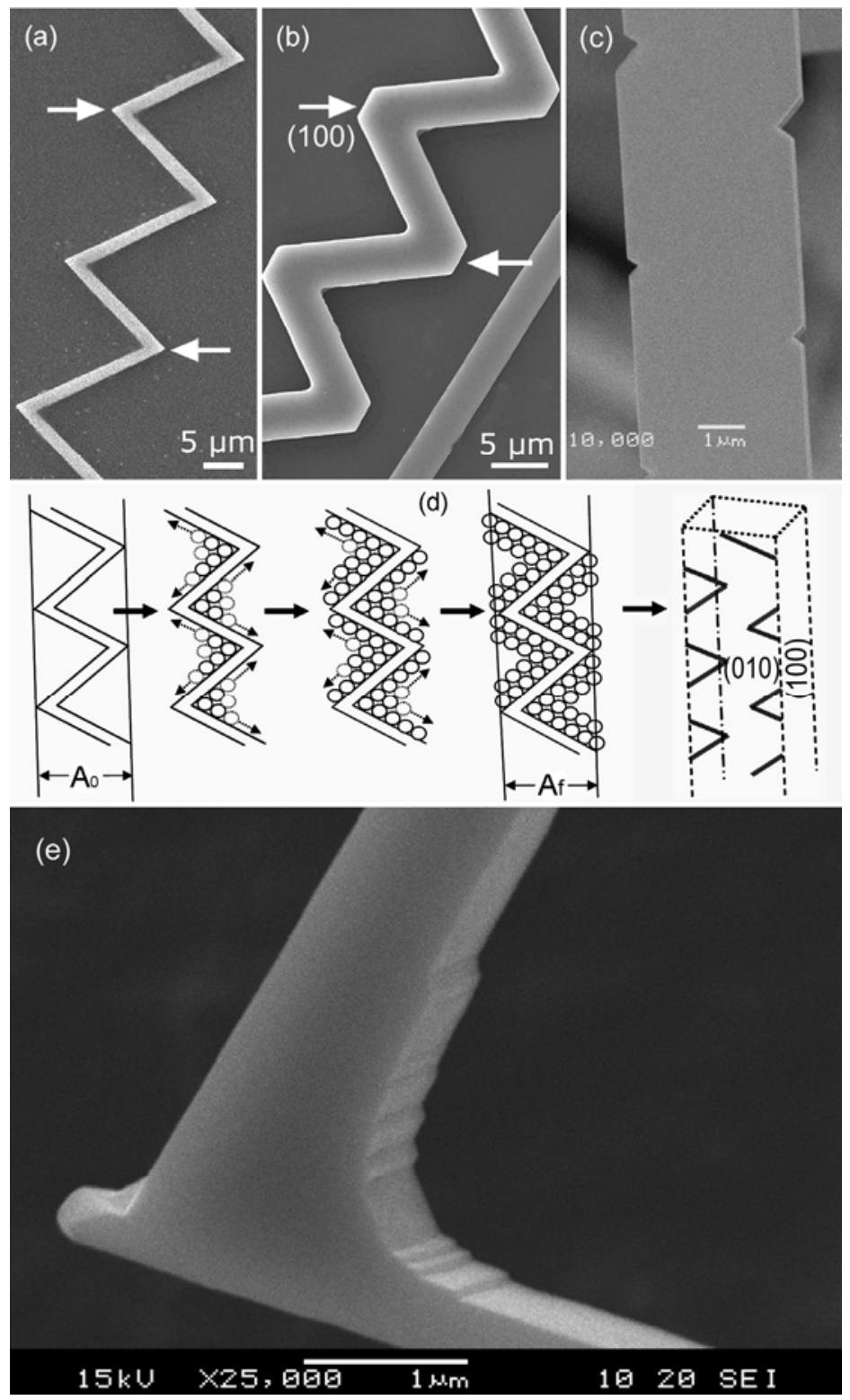

Fig. 15. SEM images of zigzags arranged (a-c) in order to show the evolution process, in (a) the zigzag has narrow slab-like morphology and sharp corners, after epitaxial growth, in (b), (c), the zigzag shows increased width and new \pm (100) facets present, schematic illustration for the morphological evolution (d), the vapor species favor deposition at the V-like slots and it results in some atom steps. 


\section{Concluding remarks}

The directional growth of well-faceted $\mathrm{ZnO}$ microfibers along different axes could be realized by catalyzing growth. The characterization of the fibers by optical and photoluminescence microscopy showed that the outer facets of the crystalline fibers provide excellent mirror-like surfaces for guiding light propagation along the fiber stem as well as the periodic junction-prisms. The structure-related optical properties of the fibers can be fully explained by a micro-structural model. The model explains several optical properties, such as luminance decreasing at the junction-prisms caused by refraction and total or partial reflection of light, as well as luminance enhancement at the junction-prisms related to waveguiding of the green emission along the $\mathrm{ZnO}$ fibers. Further integration of the $\mathrm{ZnO}$ junction-prisms into micro-devices could provide micro-scale modulation for light with different wavelengths. Such capability makes such fibers potentially suitable for enhanced light-illumination arrays. Reproducibly high-yield growth of $\mathrm{SnO}_{2}$ zigzag nanofibers was achieved via controlling the reactant vapor concentration. The formation of the zigzag fiber based on the pre-growing nanobelt is suggested to be in a two-step process: the first is frame growth, which is accomplished through repeated orienting along equivalent directions; the second is lateral epitaxy, which thickens a fiber and results in well-faceted morphology. It is note that the present of intrinsic equivalent directions and the oscillation of external growth kinetics are key roles for producing zigzag structures. The elucidation of the growth mechanism should provide a fully controlled route for reproducibly high-yield growth of zigzag fibers of $\mathrm{SnO}_{2}$ and give some valuable hints to synthesis other zigzag fibers. This well-faceted zigzag fiber could be studied as optical waveguide in its periodic structure and gas sensor component. These results have shown that the optimization of surfaces, boundaries, and interfaces in 1D meso-scale materials with well-faceted structures plays an important role in furthering the application of these materials.

\section{Acknowledgement}

We thank Dr. L. Pu, Dr. M. K. Lee, Prof. C. Tien and Prof. L. J. Chang for helpful discussions. L. S. Huang is truly grateful for financial support from NSFC (No. 60606020) and NCKU's "Aim for the Top University Project".

\section{References}

[1] A. P. Alivisatos, "Semiconductor clusters, nanocrystals, and quantum dots" Science 271, 933-937 (1996).

[2] A. D. Yoffe, "Semiconductor quantum dots and related systems-electronic, optical, luminescence and related properties of low dimensional systems" Advances In Physics 50, 1-208 (2001).

[3] Z. L. Wang, "ZnO nanowire and nanobelt platform for nanotechnology" Mater. Sci. Eng. R. 64, 33-71 (2009).

[4] H. Joyce, Q. Gao, H. Tan, Jagadish, C. "Phase perfection in zinc blende and wurtzite IIIV nanowires using basic growth parameters" Nano Lett.10, 908- 915 (2010).

[5] B. M. Wong, F. Lonard, Q. M. Li, T. George "Nanoscale Effects on Heterojunction Electron Gases in GaN/AlGaN Core/Shell Nanowires" Nano Lett. 11, 3074-3079 (2011). 
[6] X. F. Qiu, C. Burda, R. L. Fu, L. Pu, H. Y. Chen, J. J. Zhu, “Heterostructured $\mathrm{Bi}_{2} \mathrm{Se}_{3}$ nanowires with periodic phase boundaries" J. Am. Chem. Soc. 126, 16276-16277 (2004).

[7] L. Manna, D. J. Milliron, A. Meisel, E. C. Scher, A. P. Alivisatos, “Controlled growth of tetrapod-branched inorganic nanocrystals" Nat. Mater. 2, 382-385 (2003).

[8] L. S. Huang, S. Wright, S. Yang, D. Z. Shen, B. X. Gu, Y. W. Du, “ZnO well-faceted fibers with periodic junctions" J. Phys. Chem. B. 108, 19901-19903 (2004).

[9] M. H. Huang, S. Mao, H. Feick, H. Yan, Y. Wu, H. Kind, E. Weber, R. Russo, P. Yang, "Room-temperature ultraviolet nanowire nanolasers" Science 292, 1897-1899 (2001).

[10] J. Johnson, H. J. Choi, K. P. Knutsen, R. D. Schaller, P. Yang, R. J. Saykally, “Single gallium nitride nanowire lasers" Nat. Mater. 1, 106-110 (2002).

[11] M. Law, D. Sirbuly, J. Johnson, J. Goldberger, R. Saykally, P. Yang, “Ultralong nanoribbon waveguides for sub-wavelength photonics integration" Science 305,1269-1271 (2004).

[12] C. J. Barrelet, A. B. Greytak, C. M. Lieber, "Nanowire photonic circuit elements," Nano Lett. 4, 1981-1985 (2004).

[13] X. G. Peng, L. Manna, W. D. Yang, J. Wickham, E. C. Scher, A. Kadavanich, A. P. Alivisatos, "Shape control of CdSe nanocrystals," Nature 404, 59-61 (2000).

[14] R. Jin, Y. C. Cao, E. Hao, G. S. Métraux, G. C. Schatz, C. A. Mirkin, “Controlling anisotropic nanoparticle growth through plasmon excitation," Nature 425, 487-490 (2003).

[15] Z. R. Tian, J. Voigt, J. Liu, B. Mckenzie, M. McDermott, M. Rodriguez, H. Konishi, H. F. $\mathrm{Xu}$, "Complex and oriented ZnO nanostructures," Nat. Mater. 2, 821-826 (2003).

[16] T. Schlli, R. Daudin, G. Renaud, A. Vaysset, O. Geaymond, A. Pasturel, "Substrate enhanced supercooling in AuSi eutectic droplets" Nature 464, 1174- 1177 (2010).

[17] M. Tabuchi, A. M. Y. Ohtake, Y. Takeda, "X-ray CTR scattering measurement to investigate the formation process of InP/GaInAs interface" J. Phys.: Conf. Ser. 83, 012031 (2007).

[18] M. Verheijen, R. Algra, M. Borgstrm, G. Immink, E. Sourty, W. van Enckevort, E. Vlieg, E. Bakkers, "Three dimensional morphology of GaP-GaAs nanowires revealed by transmission electron microscopy tomography" Nano Lett. 7, 3051-3055 (2007).

[19] D. E. Perea, N. Li, R. M. Dickerson, A. Misra, S. T. Picraux, “Controlling Heterojunction Abruptness in VLS-Grown Semiconductor Nanowires via in situ Catalyst Alloying" Nano Lett. 11, 3117-3122 (2011).

[20] O. Shpyrko, R. Streitel, V. Balagurusamy, A. Grigoriev, M. Deutsch, B. Ocko, M. Meron, B. Lin, P. Pershan, "Surface crystallization in a liquid AuSi alloy" Science 313, 77-80 (2006).

[21] D. C. Look, "Recent advances in ZnO materials and devices" Mat. Sci. Eng. B. 80, 383387 (2001).

[22] D. M. Bagnall, Y. F. Chen, Z. Zhu, S. Koyama, M. Y. Shen, T. Goto, “Optically pumped lasing of ZnO at room temperature" Appl. Phys. Lett. 70, 2230-2232 (1997).

[23] P. M. Martin, M. S. Good, J. W. Johnston, L. J. Bond, S. L. Crawford, "Piezoelectric films for 100-MHz ultrasonic transducers" Thin Solid Films 379, 253-258 (2000).

[24] J. Q. Xu, Q. Y. Pan, Y. A. Shun , Z. Z. Tian, “Grain size control and gas properties of ZnO gas sensors" Sensors Actuators B. 66, 277-279 (2000). 
[25] M. H. Huang, S. Mao, H. Feick, H. Q. Yan, Y. Y. Wu, H. Kind, E. Weber, R. Russo, P. D. Yang, "Room-temperature ultraviolet nanowire nanolasers" Science 292, 1897-1899 (2001).

[26] J. H. Song, Y. Zhang, , C. Xu, W. Z. Wu , Z. L. Wang, "Polar Charges Induced Electric Hysteresis of ZnO Nano/Microwire for Fast Data Storage" Nano Lett. 11, 2829_ 2834 (2011).

[27] H. Kind, H. Yan, B. Messer, M. Law, P. Yang, “Nanowire ultraviolet photodetectors and optical switches" Adv. Mater. 14, 158-160 (2002).

[28] Y. Wu, H. Yan, P. Yang, "Semiconductor nanowire array: potential substrates for photocatalysis and photovoltaics" Top. Catal. 19, 197-202 (2002).

[29] G. Gordillo, "New materials used as optical window in thin film solar cells" Surf. Rev. Lett. 9, 1675-1680 (2002).

[30] W. I. Park, G. C. Yi, J. W. Kim, S. M. Park, "Schottky nanocontacts on ZnO nanorod arrays" Appl. Phys. Lett. 82, 4358-4360 (2003).

[31] A. F. Yu, H. Y. Li, H. Y. Tang, T. J. Liu, P. Jiang, Z. L. Wang, “Vertically integrated nanogenerator based on ZnO nanowire arrays" Physica Status Solidi. 5, 162-164 (2011).

[32] N. Audebrand, J. P. Auffredic, D. Louer, "X-ray diffraction study of the early stages of the growth of nanoscale zinc oxide crystallites obtained from thermal decomposition of four precursors" Chem. Mater. 10, 2450-2461 (1998).

[33] A. M. Morales, C. M. Lieber, "A laser ablation method for the synthesis of crystalline semiconductor nanowires" Science 279, 208-211 (1998).

[34] Z. W. Pan, Z. R. Dai, Z. L. Wang, "Nanobelts of semiconducting oxides" Science 291, 1947-1949 (2001).

[35] M. H. Huang, Y. Y. Wu, H. Feick, N. Tran, E. Weber, P. D. Yang, “Catalytic growth of zinc oxide nanowires by vapor transport" Adv. Mater. 13, 113-116 (2001).

[36] Y. G. Wei, W. Z. Wu, R. D. Guo, J. Yuan, D. Suman, Z. L. Wang, “Wafer-Scale HighThroughput Ordered Growth of Vertically Aligned ZnO Nanowire Arrays" Nano Lett. 11, 2829-2834 (2011).

[37] G. D. Yuan, W. J. Zhang, S. T. Lee "p-Type ZnO Nanowire Arrays" Nano Lett. 8, 25912597 (2008).

[38] Y. Li, D. W. Meng, L. D. Zhang, F. Phillip, “Ordered semiconductor ZnO nanowire arrays and their photoluminescence properties" Appl. Phys. Lett. 76, 2011-2013 (2000).

[39] D. S. Boyle, K. Govender, P. O'Brien, “Novel low temperature solution deposition of perpendicularly orientated rods of $\mathrm{ZnO}$ : substrate effects and evidence of the importance of counter-ions in the control of crystallite growth" Chem. Commun. 1, 80-81 (2002).

[40] L. Vayssieres, K. Keis, S. Lindquist, A. Hagfeldt, "Purpose-built anisotropic metal oxide material: 3D highly oriented microrod array of ZnO" J. Phys. Chem. B 105, 33503352 (2001).

[41] L. Vayssieres, "Growth of arrayed nanorods and nanowires of $\mathrm{ZnO}$ from aqueous solutions" Adv. Mater. 15, 464-466 (2003).

[42] L. Guo, S. H. Yang, C. L. Yang, J. N. Wang, W. K. Ge, “Synthesis and Characterization of Poly (vinylpyrrolidone)-Modified Zinc Oxide Nanoparticles" Chem. Mater. 12, 2268-2274, (2000). 
[43] Z. R. Tian, J. A. Voigt, J. Liu, B. Mckenzie, M. J. Mcdermott, “Biomimetic arrays of oriented helical ZnO nanorods and columns" J. Am. Chem. Soc. 124, 12954-12955 (2002).

[44] L. E. Greene, M. Law, D. H. Tan, P. D. Yang “General Route to Vertical ZnO Nanowire Arrays Using Textured ZnO Seeds" Nano Lett. 5, 1231-1236 (2005).

[45] X. Y. Kong, Y. Ding, R. S. Yang, Z. L. Wang, "Single-crystal nanorings formed by epitaxial self-coiling of polar nanobelts" Science 303, 1348-1351 (2004).

[46] Y. J. Su, Y. J. Zhou, L. S. Huang, Y. F. Liu, S. Z. Shi, Y. N. Lv, “The morphology evolution mechanism of $\mathrm{ZnO}$ quasi-one-dimensional nanostructures" J. Inor. Mater. 24, 6 (2009).

[47] C. A. Arguello, D. L. Rousseau, S. P. S. Porto, "First-Order Raman Effect in WurtziteType Crystals" Phys. Rev. 181, 1351-1363 (1969).

[48] T. C. Damen, S. P. S. Porto, B. Tell, "Raman Effect in Zinc Oxide” Phys. Rev. 142, 570574 (1966).

[49] L. E. Greene, M. Law, J. Goldberger, F. Kim, J. C. Johnson, Y. F. Zhang, R. J. Saykally, P. D. Yang, "Low-temperature wafer-scale production of $\mathrm{ZnO}$ nanowire arrays" Angew. Chem. Int. Ed. 42, 3031-3034 (2003).

[50] A.Van Dijken, E. A. Meulenkamp, D.Vanmaekelbergh, A. Meijerink, "Identification of the transition responsible for the visible emission in $\mathrm{ZnO}$ using quantum size effects" J. Lumin. 90, 123-128 (2000).

[51] L. S. Huang, L. Pu, Y. Shi, R. Zhang, B. X. Gu, Y. W. Du, Y. D. Zheng, "Light propagation tuned by periodic junction-prisms within well-faceted $\mathrm{ZnO}$ fibers" Opt. Express, 13, 5263-5269, (2005).

[52] Z. M. Jarzebski, J. P. Marton, "Physical properties of SnO2 materials" J. Electrochem. Soc. 123, 199c-205c (1976).

[53] Z. R. Dai, Z. W. Pan, Z. L. Wang, “Ultra-long single crystalline nanoribbons of tin oxide" Solid State Commun. 118, 351-354 (2001).

[54] C. Y. Wen, J. Tersoff, K. Hillerich, M. C. Reuter, J. H. Park, S. Kodambaka, E. A. Stach1, and F. M. Ross "Periodically Changing Morphology of the Growth Interface in Si, Ge, and GaP Nanowires" Phys. Rev. Lett. 107, 025503 (2011).

[55] J. Q. Hu, Y. Bando, D. Golberg, "Self-catalyst growth and optical properties of novel SnO2 fishbone-like nanoribbons" Chem. Phys. Lett. 372, 758-762 (2003).

[56] A. Beltran, J. Andres, E. Longo, E. R. Leite, “Thermodynamic argument about SnO2 nanoribbon growth" Appl. Phys. Lett. 83, 635-637 (2003).

[57] B. Thiel, R. Helbig, " Growth of $\mathrm{SnO} 2$ single crystals by a vapour phase reaction method" J. Crystal Growth 32, 259-264 (1976).

[58] L. S. Huang, L. Pu, Y. Shi, R. Zhang, B. X. Gu, Y. W. Du, S. Wright, “Controlled growth of well-faceted zigzag tin oxide mesostructures" Appl. Phys. Lett., 87, 163124 (2005). 


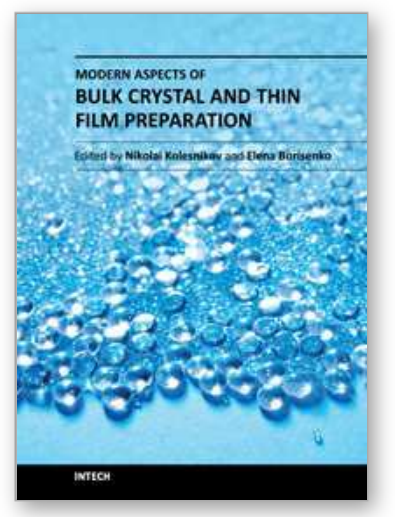

\author{
Modern Aspects of Bulk Crystal and Thin Film Preparation \\ Edited by Dr. Nikolai Kolesnikov
}

ISBN 978-953-307-610-2

Hard cover, 608 pages

Publisher InTech

Published online 13, January, 2012

Published in print edition January, 2012

In modern research and development, materials manufacturing crystal growth is known as a way to solve a wide range of technological tasks in the fabrication of materials with preset properties. This book allows a reader to gain insight into selected aspects of the field, including growth of bulk inorganic crystals, preparation of thin films, low-dimensional structures, crystallization of proteins, and other organic compounds.

\title{
How to reference
}

In order to correctly reference this scholarly work, feel free to copy and paste the following:

Lisheng Huang,Yinjie Su and Wanchuan Chen (2012). One-Dimensional Meso-Structures: The Growth and the Interfaces, Modern Aspects of Bulk Crystal and Thin Film Preparation, Dr. Nikolai Kolesnikov (Ed.), ISBN: 978-953-307-610-2, InTech, Available from: http://www.intechopen.com/books/modern-aspects-of-bulkcrystal-and-thin-film-preparation/one-dimensional-meso-structures-the-growth-and-the-interfaces

\section{INTECH}

open science | open minds

\author{
InTech Europe \\ University Campus STeP Ri \\ Slavka Krautzeka 83/A \\ 51000 Rijeka, Croatia \\ Phone: +385 (51) 770447 \\ Fax: +385 (51) 686166 \\ www.intechopen.com
}

\author{
InTech China \\ Unit 405, Office Block, Hotel Equatorial Shanghai \\ No.65, Yan An Road (West), Shanghai, 200040, China \\ 中国上海市延安西路65号上海国际贵都大饭店办公楼 405 单元 \\ Phone: +86-21-62489820 \\ Fax: +86-21-62489821
}


(C) 2012 The Author(s). Licensee IntechOpen. This is an open access article distributed under the terms of the Creative Commons Attribution 3.0 License, which permits unrestricted use, distribution, and reproduction in any medium, provided the original work is properly cited. 